\title{
Uso inadecuado de tromboprofilaxis en pacientes postquirúrgicos en cirugía general
}

\author{
Improper use of tromboprofilaxis in post-surgical patients in general surgery \\ Eric Misael Saucedo Moreno, ${ }^{*}$ Mariana Delgado Nava, ${ }^{\ddagger}$ Piero Carvallo Maiocco $\$$
}

\section{Resumen}

Antecedentes: Un paciente que se somete a un procedimiento quirúrgico tiene $20 \%$ más riesgo para presentar trombosis venosa profunda; el riesgo aumenta en $30 \%$ cuando hay ausencia de profilaxis óptima. Material y métodos: Estudio retrospectivo, transversal, descriptivo, en un periodo que abarca de 2016 a 2017. A todos se les aplicó puntuación de Caprini. La terapia tromboprofiláctica administrada a cada paciente fue analizada y comparada para estimar el cumplimiento. Resultados: Hubo un total de 100 pacientes. El número de pacientes en riesgo bajo, moderado, alto y muy alto, según la puntuación de Caprini, fue 2, 15, 31 y $52 \%$, respectivamente. Se dividió en dos grupos: profilaxis adecuada (58\%) y profilaxis inadecuada (42\%). EI $88 \%$ de pacientes con un muy alto riesgo de trombosis no presentó anticoagulación adecuada, pero el $16 \%$ de pacientes presentó trombosis venosa profunda (TVP), con una diferencia significativa de $p \leq 0.000$. Conclusiones: El estudio triunfó en la estimación de la prevalencia de episodios de trombosis venosa profunda, debido a una deficiencia en la profilaxis antitrombótica; aunque no representó aumento en la mortalidad, sí se observó un aumento en la morbilidad postquirúrgica.

Palabras clave: Tromboprofilaxis, trombosis venosa, cirugía.

\begin{abstract}
Background: A patient undergoing a surgical procedure has a $20 \%$ higher risk for VTE and the risk increases by $30 \%$ when there is an absence of optimal prophylaxis. Material and methods: Retrospective, transversal, descriptive study in a period of 2016-2017. Caprini Score was applied. The thromboprophylactic therapy administered to each patient was analyzed and compared with this guideline to estimate compliance. Results: A total of 100 patients. The number of patients at low, moderate, high and very high risk, according to the Caprini score, was $2,15,31$ and $52 \%$, respectively. It was divided into two groups, adequate prophylaxis $(58 \%)$ and inadequate prophylaxis $(42 \%), 88 \%$ of these patients with very high risk of thrombosis, did not present adequate anticoagulation, at the end $16 \%$ patients had DVT with significant difference $p \leq 0.000$. Conclusions: The study triumphed in the estimation of the prevalence of episodes of deep vein thrombosis due to a deficiency in the antithrombotic prophylaxis, although it did not represent an increase in the mortality, if an increase in the postoperative morbidity is observed.
\end{abstract}

Keywords: Thromboprophylaxis, venous thromboembolism, surgery.

diagnosticada, culminando en complicaciones crónicas y mortalidad.

El tromboembolismo venoso (TEV) se ha convertido en la tercera causa de muerte cardiovascular después del infarto agudo del miocardio (IAM) y de la enfermedad vascular cerebral (EVC), con una incidencia de 100 casos por cada 100,000 . Los estudios de autopsia demuestran un gran

La trombosis venosa es una enfermedad que abarca tanto a la trombosis venosa profunda (TVP) como a la embolia pulmonar (EP). Con aptitud para producir morbilidad y mortalidad, la TVP representa un problema de salud mundial. A menudo, permanece asintomática o sub-

Hospital Ángeles Mocel de la Ciudad de México. México.

* Facultad Mexicana de Medicina Universidad La Salle. Servicio de Cirugía General.

* Residente del primer año del Servicio de Medicina Interna. Facultad Mexicana de Medicina Universidad La Salle.

$\S$ Médico pasante del servicio social del Servicio de Cirugía General. Correspondencia:

Correo electrónico: eric.saucedo.m@gmail.com Universidad Anáhuac.

Aceptado: 17-07-2019.

www.medigraphic.com/actamedica 
número de eventos silentes, reportando dos millones de casos de TVP y hasta 200 mil muertes por EP anualmente. ${ }^{1}$

El paciente en alto riesgo incluye aquéllos que han sido sometidos a procedimientos ortopédicos importantes, en el que 50 a $60 \%$ de los pacientes tiene propensión a desarrollar trombosis venosa profunda. Aunque la profilaxis en procedimientos ortopédicos tiene la debida importancia, el riesgo asociado con procedimientos en cirugía general ha sido subestimado en todo el mundo. Un paciente sometido a procedimiento quirúrgico tiene $20 \%$ más riesgo para TEV, y el riesgo aumenta en 30\% cuando hay ausencia de profilaxis óptima. El riesgo asociado con la TEV en cirugía general varía entre 10 y 50\%, dependiendo del tipo de cirugía y de los factores de riesgo del paciente..$^{2-4}$

\section{MATERIAL Y MÉTODOS}

Estudio retrospectivo, transversal, descriptivo, con datos de expedientes de pacientes que fueron operados en un periodo de 2016 a 2017, en un hospital de tercer nivel del sector privado en la Ciudad de México. Se incluyeron a sujetos mayores de 18 años de edad que hubieran sido sometidos a cirugía por el Servicio de Cirugía General. Se excluyeron a pacientes embarazadas, a pacientes admitidos para tratamiento de trombosis venosa profunda concomitante y a aquéllos que se les realizó procedimientos quirúrgicos con anestesia local.

Un total de 100 sujetos estaban matriculados dentro del hospital durante el periodo de estudio; sus datos demográficos y datos quirúrgicos fueron recogidos usando un formulario de datos validados internamente. La puntuación de Caprini se utilizó para estratificar a los pacientes de acuerdo con el riesgo de trombosis venosa profunda en bajo, moderado, alto riesgo y muy alto riesgo. La puntuación total de riesgo de TVP fue asignada a cada paciente y se estimó a través de la sumatoria de los puntos individuales. Condiciones tales como sangrado activo, hemorragia grave previa, trastornos hemorrágicos conocidos o no tratados, insuficiencia renal o hepática severa, trombocitopenia, accidente cerebrovascular agudo, hipertensión arterial sistémica no controlada, uso concomitante de anticoagulantes, terapia antiplaquetaria o medicamentos trombolíticos fueron considerados como factores de riesgo para sangrado, según las recomendaciones de la American College of Clinical Pharmacy (ACCP). La presencia de estas condiciones fue asumida como contraindicaciones para la administración de profilaxis farmacológica.

La terapia tromboprofiláctica administrada a cada paciente fue analizada y comparada para estimar el cumplimiento. Se dividieron a los participantes en dos grupos: profilaxis adecuada y con mala profilaxis. Definimos profilaxis adecuada como aquellos pacientes a quienes se les dio el tratamiento recomendado por la guía de la ACCP, basado en el riesgo tromboembólico calculado con el test de Caprini. Se consideró profilaxis inadecuada como aquéllos a quienes no se les dio el tratamiento recomendado por la guía de la ACCP, de acuerdo con el riesgo tromboembólico. Las variables con distribuciones paramétricas se analizaron con la prueba t de Student y se expresan como media \pm desviación estándar (DE); por su parte, aquéllos con distribuciones no paramétricas fueron analizados con la prueba $U$ de Mann-Whitney y se presentaron como medianas y rangos intercuartiles (IQRs). La prueba exacta de $\chi^{2}$ o Fisher se utilizó para datos categóricos. Se utilizó el valor menor a 0.05 para una p significativa con IC del 95\%. El análisis estadístico de los datos se realizó mediante SPSS (versión 20.0) para Windows.

\section{RESULTADOS}

De acuerdo con los criterios predeterminados se incluyeron en el estudio un total de 100 pacientes. En la Tabla 1, se hace referencia a las variables generales de los participantes. El $46 \%$ de los pacientes fue operado mediante cirugía

\begin{tabular}{|lc|}
\multicolumn{2}{|c|}{ Tabla 1: Frecuencia de variables generales. } \\
\hline Variable & Valores \\
\hline Edad (DE) & \\
Sexo (F/M) & $51.43(18.402)$ \\
Cirugía laparoscópica & $54 / 46$ \\
Sí & $(\%)$ \\
No & 46 \\
Cáncer & 54 \\
Sí & \\
No & 19 \\
Nivel de riesgo & 81 \\
Bajo & \\
Moderado & 2 \\
Alto & 15 \\
Muy alto & 31 \\
Profilaxis & 52 \\
Medias TED & 18 \\
Deambulación & 7 \\
Medias TED y deambulación & 62 \\
Enoxaparina y medias TED & 13 \\
Profilaxis adecuada & \\
Sí & 58 \\
No & 42 \\
Se confirmó diagnóstico & \\
Sí & 7 \\
No & 93 \\
Muerte & \\
Sí & 2 \\
No & 98 \\
&
\end{tabular}


laparoscópica y el 54\% con cirugía abierta. La presencia de cáncer fue de 19\%. Para el análisis estadístico se dividió a los participantes en dos grupos: grupo 1 , que representa aquéllos con profilaxis adecuada (58\%) y, grupo 2, que fueron los pacientes con profilaxis no adecuada (42\%). De la muestra total, el $46 \%$ fue de varones y el restante de mujeres, lo cual no mostró una diferencia significativa en la distribución del género. La edad media de la muestra total se determinó en $51.43 \pm 18.4$ años. La diferencia en la distribución de la edad con respecto al uso de profilaxis adecuada tampoco fue significativa. La media del test Caprini fue de $5.53( \pm 3.68)$ puntos. El número de pacientes en riesgo bajo, moderado, alto y muy alto fue de $2,15,31$ y $52 \%$, respectivamente, con una diferencia significativa entre grupos $\mathrm{p} \leq 0.000$.

Los pacientes que se diagnosticaron con TVP para el grupo de profilaxis adecuada fueron de $5.1 \%$ en comparación con el grupo 2, con diagnóstico en $16 \%$, y una diferencia estadísticamente significativa para morbilidad $(p \leq 0.000)$. Dos pacientes murieron a consecuencia de TVP, uno por cada grupo, sin que esto representara significancia estadística para el aumento de la mortalidad $(p=0.818)$ (Tabla 2). Observamos que las medidas antitrombóticas más recurridas fueron el uso de medias de compresión TED y deambulación temprana, sin importar el riesgo tromboembólico en el que se encontraban.

\section{DISCUSIÓN}

Actualmente, la profilaxis de TVP en procedimientos de mínima invasión se ha vuelto un tema de relevancia. De acuerdo con la estratificación del riesgo de tromboembolismo de la última edición de la guía de la ACCP, el riesgo de TVP es bajo y con menos del 10\% sin anticoagulación. La Asociación Europea de Cirugía Laparoscópica ha recomendado la profilaxis mecánica en todos los procedimientos laparoscópicos prolongados, ${ }^{2}$ porque no se asocia con el aumento en el riesgo de sangrado. Estos dispositivos incluyen medias de compresión elástica y dispositivos de compresión intermitente. Las medidas mecánicas pueden ser la única opción adecuada en pacientes con alto riesgo de hemorragia, como ancianos que presentan de 10 a 20 veces mayor riesgo de presentar TEV, debido a una creciente prevalencia de enfermedades crónicas que contribuyen a esta condición, además de ser frecuentemente hospitalizados por enfermedades agudas y por cirugías urgentes o electivas, en las que muchas veces la recuperación de la movilidad es más lenta. No obstante, estos dispositivos mecánicos a menudo no se utilizan de forma correcta, por lo que su empleo en la práctica puede dar resultados diferentes. ${ }^{1}$

Un metaanálisis para la adherencia a profilaxis mecánica de 221 estudios, con una población de 3,080 pacientes, demostró que la adherencia a las medias de compresión intermitente fue de 75\% (rango 40-89\%), identificando que las causas de no adherencia se debían a: 1) dificultad para dormir, 2) incomodidad, 3) los dispositivos eran demasiado calientes y 4) no eran reemplazados de forma adecuada por enfermería. ${ }^{5}$ En nuestro estudio, a los pacientes de manera rutinaria se les colocan medias de compresión y deambulación temprana, encontrando

Tabla 2: Tabla comparativa entre grupos con profilaxis adecuada y no adecuada.

\begin{tabular}{lccc} 
& Profilaxis adecuada $(\mathrm{n}=58)$ & Profilaxis no adecuada $(\mathrm{n}=42)$ & $\mathrm{p}$ \\
\hline Edad & $45.38(18.022)$ & $59.79(15.58)$ & 0.312 \\
Sexo F/M & $30 / 28$ & $24 / 18$ & 0.592 \\
Cirugía laparoscópica & $25(43 \%)$ & $21(50 \%)$ & 0.546 \\
Cáncer & $9(15 \%)$ & $10(23 \%)$ & 0.297 \\
\hline Nivel de riesgo & & & \\
Bajo & $2(3 \%)$ & 0 & \\
Moderado & $15(25 \%)$ & $5(11 \%)$ \\
Alto riesgo & $26(44 \%)$ & $37(88 \%)$ \\
Muy alto riesgo & $15(25 \%)$ & & \\
\hline Profilaxis & & $10(23 \%)$ \\
Medias TED & $8(13 \%)$ & $6(14 \%)$ \\
Deambulación & $1(1.7 \%)$ & $26(61 \%)$ \\
Medias TED y deambulación & $36(62 \%)$ & 0 \\
Enoxaparina y medias TED & $13(22 \%)$ & $7(16 \%)$ \\
\hline Se confirmó diagnóstico & $3(5.1 \%)$ & $1(2.3 \%)$ \\
Fallecimientos & $1(1.7 \%)$ & 0.001
\end{tabular}


un abuso en el uso de estas medidas como única medida antitrombótica, por lo que no cumple la profilaxis adecuada en un mínimo de $26 \%$ de los pacientes estudiados $(p=0.000)$.

Una evaluación cuidadosa del riesgo de trombosis venosa debe ser una práctica habitual. Se han presentado modelos de evaluación de riesgo formal; sin embargo, éstos no son utilizados de forma rutinaria, ya que no han sido adecuadamente validados. Un enfoque estratifica a los pacientes quirúrgicos, asignándolos a uno de los cuatro niveles de riesgo de TEV, basado en el tipo de operación (menor y mayor), edad ( $<40$ años, 40-60 años y $>60$ años) y la presencia de factores de riesgo adicionales, siendo todos elementos poco objetivos. ${ }^{2,4}$

La escala de Caprini es simple y distingue de manera adecuada el riesgo tromboembólico; también ha sido validada en cirugía general, vascular, urológica y plástica. Recomendaciones del ACCP, con base en diferentes estudios, han demostrado que el puntaje de Caprini representa una estrategia profiláctica basada en el riesgo específico para cada paciente. Según los resultados pueden distinguirse cuatro categorías de riesgo en ausencia de profilaxis: riesgo bajo, TVP $<0.5 \%$ (Caprini 1); riesgo moderado, TVP 1.5\% (Caprini 2); riesgo alto, TVP 3\% (Caprini 3-4); riesgo muy elevado, TVP 6\% (Caprini $\geq 5$ ). ${ }^{6,7}$ Una vez clasificado se recomienda que para pacientes con un riesgo bajo puede no utilizarse profilaxis o profilaxis mecánica; para pacientes con riesgo moderado se recomienda de forma rutinaria profilaxis mecánica; para pacientes con riesgo alto se recomienda el inicio de tromboprofilaxis farmacológica con heparinas de bajo peso molecular (HBPM) o heparina no fraccionada (HNF) y mecánica; para pacientes con riesgo elevado se recomienda iniciar tratamiento farmacológico con HPBM y HNF, además de profilaxis mecánica con medias de compresión neumática intermitente o medias antitrombosis y también en pacientes sin contraindicación con fondaparinux, o aspirina a baja dosis. En nuestro estudio fueron clasificados con base en el test de Caprini, de los cuales 52\% presentó un muy alto riesgo de trombosis venosa profunda; a su vez, 88\% (37) de estos pacientes no presentó anticoagulación adecuada, al final 16\% (siete pacientes) presentó TVP, lo cual clínicamente es un porcentaje enorme de casos de TVP por ausencia de clasificación y manejo profiláctico adecuado.

Las heparinas de bajo peso molecular se han convertido en el tratamiento profiláctico de elección para tromboembolismo venoso en pacientes operados en el Servicio de Cirugía General, gracias a su capacidad de reducir la incidencia de TVP asintomática, lo que también contribuye a la disminución en el riesgo de TEP o la muerte. ${ }^{8}$
En un metaanálisis que comparó la profilaxis de HBPM en comparación con placebo o no tratamiento, se observó una reducción estadísticamente significativa del $72 \%$ para el riesgo de TVP en el grupo de HBPM. También demostró una disminución de $75 \%$ en el riesgo para TEP y $71 \%$ para TEV; asimismo, tuvo un aumento de hemorragia del 103\% para hemorragia mayor, 106\% para hemorragia en general, $88 \%$ para hematoma en herida y $53 \%$ en el número de pacientes que requirieron transfusión postoperatoria en el grupo tratado con heparina.

En comparación de HBPM con HNF, se redujo 10\% el riesgo de TVP en el grupo de HBPM, siendo este resultado no estadísticamente significativo. Asimismo, se redujo en $12 \%$ los eventos de TEP y se disminuyó el riesgo de hemorragia mayor y de hematoma en el grupo de HBPM; sin embargo, esto no fue significativo. Se concluyó en este estudio que las HBPM prácticamente presentan la misma efectividad que la HNF, si bien su uso es mucho más sencillo, por lo que se considera la referencia de tratamiento. ${ }^{8}$

En un estudio se comparó fondaparinux con HBPM en pacientes sometidos a cirugía abdominal mayor. Las tasas de TEV en los grupos fondaparinux y dalteparina fueron de 4.6 y $6.1 \%$, respectivamente. Las tasas de sangrado mayor no fueron significativamente diferentes (3.4 y $2.4 \%$ ). Para aquellos pacientes sometidos a cirugía de cáncer abdominal, la incidencia de TEV fue significativamente menor en los receptores de fondaparinux en comparación con los de dalteparina (4.7 vs $7.7 \%)^{1}$

El uso de ASA como alternativa farmacológica a otros anticoagulantes en pacientes sometidos a cirugías mayores fue recomendado por la ACCP en 2012. Dos estudios han demostrado evidencia de su eficacia en la profilaxis antitrombótica. En uso como monoterapia en comparación con placebo se observó una disminución del riesgo de TVP de $23 \%$, y para TEP de $6 \%$; sin embargo, su eficacia es incierta en comparación contra otros anticoagulantes. ${ }^{9}$

Es conocido que el cáncer es un factor de riesgo para TVP, lo que aumenta en general la mortalidad en los siguientes 30 días posteriores a la operación. La hipercoagulabilidad es mediada por el incremento de los niveles del factor de crecimiento endotelial (VEGF), factor tisular y la terapéutica (quimio y radioterapia). El National Comprehensive Cancer Network y la American Society of Clinical Oncology recomiendan extender la tromboprofilaxis por al menos cuatro semanas. De la misma forma, algunos ensayos controlados han demostrado que la profilaxis prolongada por cuatro semanas después de cirugía laparoscópica por cáncer colorrectal es segura, además de reducir significativamente la TVP en comparación con la primera semana de profilaxis. ${ }^{8,10}$ En nuestro estudio no hubo diferencia significativa entre los dos grupos en aqueIlos pacientes con diagnóstico de cáncer $(p=0.297)$; no 
obstante, el seguimiento que dimos en nuestro estudio no fue el suficiente como para descartar del todo la probabilidad de TVP silente o sintomática.

Una de las deficiencias de nuestro estudio es, sin duda, el número de pacientes; aunque es suficiente para obtener resultados con un gran impacto y significancia, es necesario aumentar la población y demostrar que el uso de tromboprofilaxis es inadecuado en cirugía general. Otra de las deficiencias del estudio se debe a que no pudimos realizar estudios comprobatorios de trombosis venosa profunda silente, lo cual muy probablemente podría cambiar los resultados hacia una mayor morbilidad, sin olvidarnos que el seguimiento sólo fue sobre su internamiento postquirúrgico. Desconocemos si, posterior al egreso, presentaron cuadro sintomático del mismo o, peor aún, mortalidad secundaria.

\section{CONCLUSIONES}

El estudio triunfó en determinar la estimación de la prevalencia de episodios de trombosis venosa profunda, debido a una deficiencia en la profilaxis antitrombótica. Aunque esto no representó un aumento en la mortalidad, sí se observó un aumento en la morbilidad postquirúrgica. Los médicos deben abstenerse de usar deambulación precoz como única estrategia profiláctica. La adecuación de la profilaxis de la trombosis, aunque comparable a estimaciones a nivel mundial, todavía tiene un margen de mejora a través de la planificación meticulosa y adecuada.

\section{REFERENCIAS}

1. Jaffer AK, Brotman DJ. Prevention of venous thromboembolism after surgery. Clin Geriatr Med. 2008; 24 (4): 625-639. doi:10.1016/j. cger.2008.08.001.
2. Squizzato A, Venco A. Thromboprophylaxis in day surgery. Int J Surg. 2008; 6 (Suppl. 1): S29-S30. doi:10.1016/j. ijsu.2008.12.020.

3. Zaghiyan KN, Sax HC, Miraflor E, Cossman D, Wagner W, Mirocha $J$ et al. Timing of chemical thromboprophylaxis and deep vein thrombosis in major colorectal surgery. Ann Surg. 2016; 264 (4): 632-639. doi:10.1097/SLA.0000000000001856.

4. Galante M, Languasco A, Gotta D, Bell S, Lancelotti T, Knaze V et al. Venous thromboprophylaxis in general surgery ward admissions: strategies for improvement. Int J Qual Heal Care. 2012; 24 (6): 649656. doi:10.1093/intqhe/mzs052.

5. Craigie S, Tsui JF, Agarwal A, Sandset PM, Guyatt GH, Tikkinen KA. Adherence to mechanical thromboprophylaxis after surgery: a systematic review and meta-analysis. Thromb Res. 2015; 136 (4): 723-726. doi:10.1016/j.thromres.2015.06.023.

6. National T, Forum Q, Services M. AORN guidelines for prevention of venous stasis. AORN J. 2007; 85 (3): 607. doi:10.1016/S00012092(07)60131-8.

7. Zaragoza-Delgadillo JC, Sauri-Arce JCA, Ochoa-Carrillo FJ, Fuentesdel-Toro S. Prevención de la enfermedad tromboembólica venosa en cirugía. Gac Med Mex. 2007; 143 (Suppl. 1): 35-39. doi:https:// doi.org/10.1016/S1280-4703(16)79274-7.

8. Mismetti P, Laporte S, Darmon J, Buchmüller A, Decousus H. Metaanalysis of low molecular weight heparin in the prevention of venous thromboembolism in general surgery. Br J Surg. 2001; 88 (7): 913-930. doi:10.1046/j.0007-1323.2001.01800.x.

9. Sahebally SM, Healy D, Walsh SR. Aspirin in the primary prophylaxis of venous thromboembolism in surgical patients. Surgeon. 2015; 13 (6): 348-358. doi:10.1016/j.surge.2015.05.001.

10. Borly L, Wille-Jorgensen P, Rasmussen MS. Systematic review of thromboprophylaxis in colorectal surgery-an update. Colorectal Disease. 2005; 7 (2): 122-127. doi:10.1111/j.14631318.2004.00734.x.

Conflicto de intereses: Los autores declaran no tener ningún conflicto de interés y no haber recibido patrocinio para la realización del presente trabajo. 